\title{
Overwhelming sepsis after a cat bite
}

\author{
Julie Blackburn MD¹, Émilie Tremblay MD², Catherine Tsimiklis MD FRCP1,3, \\ Benoit Thivierge MD FRCP1,3, Valéry Lavergne MD FRCP1,3
}

\section{CASE PRESENTATION}

In mid-June, a 52-year-old man presented to the emergency department with fever, chills and a rapidly progressing erythema on his right hand without regional lymphadenopathy, a few days after being bitten by his domestic cat. The patient resided in a rural area and had a history of chronic alcohol consumption. The cat lived primarily outdoors and its immunization status was unknown. Pasteurella multocida cellulitis was suspected, oral amoxicillin-clavulanate was prescribed and the patient was discharged.

Three days later, the cellulitis regressed completely but the patient sought medical attention for persisting fever and new-onset jaundice and dyspnea. On physical examination, he was hypotensive, exhibited hepatosplenomegaly and presented a centimetric necrotic ulcer on his right hand.

The patient's hematological results revealed leukopenia, thrombopenia and an elevated international normalized ratio. His biochemistry results revealed acute renal and liver failure. His chest radiograph was normal.

The patient's condition rapidly deteriorated to septic shock and multiple organ failure. He required mechanical ventilation, vasopressive support and renal replacement therapy. Intravenous piperacillin-tazobactam and vancomycin were empirically started; he was transferred to a tertiary care hospital the next day.

On arrival to the intensive care unit, a septic workup was repeated and the patient's hand ulcer was surgically debrided and tissue was sent for culture. Antibiotic treatment was switched to intravenous meropenem and vancomycin for sepsis of unknown origin. The patient developed further ascites. A percutaneous cholecystostomy was performed for suspected acalculous cholecystitis. The bile and the cloudy ascitic fluid were also cultured. Despite aggressive medical therapy, the patient's condition failed to improve during the following two days and the family decided not to further pursue active treatments. At the time of the patient's death, viral hepatitis serology and all bacterial cultures were negative.

The following day, the aerobic blood cultures showed small Gramnegative coccobacilli. What is the diagnosis?

\section{DIAGNOSIS}

After four days of incubation, the plates of aerobic blood cultures grew small grey colonies on chocolate agar and on initial sheep blood agar. The same isolate was identified in all other specimens (necrotic ulcer, bile, ascites and endotracheal secretion). Laboratory technicians were instructed to handle the plates inside the biosafety cabinet because Francisella tularensis was suspected. The Gram stain of the colonies showed the same tiny Gram-negative coccobacilli. The organism was tetramethyl-oxidase negative, weakly catalase positive and nonmotile. The isolate was sent to the provincial reference laboratory, which confirmed the identification of $F$ tularensis.

Following the postmortem diagnosis, an investigation headed by the regional public health agency revealed that the cat was still in good health and its serology for $F$ tularensis was negative. Other domestic and wild animals in the area also tested negative. There were no other cases of tularemia reported in the region during the rest of the summer. Nevertheless, informational pamphlets on tularemia were distributed to local small game hunters and warnings were published in regional newspapers to increase public awareness.

\section{DISCUSSION}

Although $F$ tularensis infects numerous vertebrates, lagomorphs and rodents are the principal reservoirs of this pathogen (1). Transmission to humans occurs most frequently either through the bite of an arthropod vector (tick, biting fly or mosquito) or through contact with a contaminated animal. Skinning, dressing and eating undercooked meat of infected animals have been associated with outbreaks of tularemia. Workers in particular occupations are also at increased risk of contracting tularemia - namely, laboratory workers, farmers, landscapers, veterinarians, hunters and meat handlers. Because it requires a very low inoculum (10 to 50 organisms), transmission through cutaneous inoculation or aerosolization is much more efficient in causing infection than gastrointestinal ingestion (1-3).

$F$ tularensis is a slow-growing and fastidious organism that requires an enriched medium with cysteine for growth such as chocolate agar, buffered charcoal-yeast extract agar and Thayer-Martin agar. It usually grows poorly on sheep blood agar, but nutritionally enriched specimens (blood and tissues) may initially permit growth on nonenriched bacteriological media, as illustrated by our case (4).

A Swedish study showed that owning a cat was an independent risk factor (OR 2.5 [95\% CI 1.5 to 4.2]) for developing tularemia (5). In the literature, more than 50 cases of tularemia secondary to a cat bite or scratch have been reported (6-11). After killing or feeding on infected prey, cats can either become infected with $F$ tularensis or can transiently carry the organisms in their mouth or claws and, thus, transmit disease to humans (8). In fact, our patient probably acquired tularemia through this transient colonization mechanism because the cat's serology remained negative.

Generally, the incubation period of tularemia is three to six days, although it can range from one day to three weeks. There are various clinical syndromes associated with tularemia: ulceroglandular, glandular, oculoglandular, pharyngeal, typhoidal and pneumonic. The ulceroglandular form is the most frequent and is characterized by an ulcer that develops at the site of entry of the pathogen and a regional lymphadenopathy in the draining territory. Systemic symptoms are usually present. Ulceroglandular syndrome by itself is rarely fatal, even without treatment. On the other hand, typhoidal tularemia is associated with a mortality rate of $30 \%$ to $60 \%$. This syndrome may result from any mode of acquisition and is difficult to diagnose because the portal of entry is often not apparent and lymphadenopathy is usually absent. The course of the illness can be quite dramatic, especially in patients with comorbid conditions or immunodeficiency (1-3).

In the present case, it appears that our patient initially presented with ulceroglandular tularemia that subsequently evolved into typhoidal tularemia due to his relative immunodeficiency conferred by a probable chronic liver disease and, potentially, to the absence of adequate antimicrobial therapy.

${ }^{1}$ Départment de microbiologie et immunologie; ${ }^{2}$ Départment de radiologie, radio-oncologie et médecine nucléaire, Faculté de médecine, Université de Montréal; ${ }^{3}$ Service de microbiologie médicale et infectiologie, Hôpital du Sacré-Coeur de Montréal, Montréal, Québec

Correspondence: Dr Julie Blackburn, Département de microbiologie et immunologie, Faculté de médecine, Université de Montréal, 2900, boulevard

Édouard-Montpetit, Pavillon Roger-Gaudry \#P-623, Montréal, Québec H3T 1J4. Telephone 514-597-1942, e-mail julie.blackburn@gmail.com 
First-line agents for the treatment of tularemia are aminoglycosides (streptomycin or gentamicin) for seven to 14 days. Alternative regimens with activity against $F$ tularensis include fluoroquinolones, doxycycline and chloramphenicol (1-3).

\section{CONCLUSION}

We presented a case of cat-bite tularemia that remained undiagnosed until blood cultures grew the causative organism. We hypothesize that the initial diagnosis of Pasteurella cellulitis responding to standard therapy and the atypical mode of transmission for tularemia misguided the differential diagnosis. Nevertheless, the presence of a necrotic ulcer on a limb should have raised the possibility of tularemia, and a high index of suspicion should always be kept when encountering a patient with a history of outdoor activities with tick or animal exposure. In the appropriate epidemiological context, empirically adding aminoglycoside, fluoroquinolone or doxycycline should be considered in a rapidly deteriorating patient.

6. Sheftel JM, Griffith JM, Leppke BA, Pantlin GC, Snippes PM, Wünschmann A. Tularaemia in Minnesota: Case report and brief epidemiology. Zoonoses Publ Health 2010;57:165-9.

7. Weinberg AN, Branda JA. Case 31-2010: A 29-year-old woman with fever after a cat bite. N Eng J Med 2010;363:1560-8.

8. Capellan J, Fong IW. Tularemia from a cat bite: Case report and review of feline-associated tularemia. Clin Infect Dis 1993;16:472-5.

9. Von Schroeder HP, McDougall EP. Ulceroglandular and pulmonary tularemia: A case resulting from a cat bite to the hand. J Hand Surg Am 1993;18A:132-4.

10. Arav-Boger R. Cat-Bite Tularemia in a seventeen-year-old girl treated with ciprofloxacin. Pediatr Infect Dis J 2000;19:583-4.

11. Yuen JC, Malotky MV. Francisella tularensis osteomyelitis of the hand following a cat bite: A case of clinical suspicion. Plast Reconstr Surg 2011;128:37-8. 


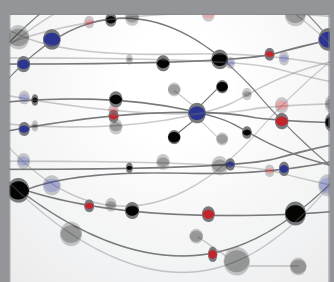

The Scientific World Journal
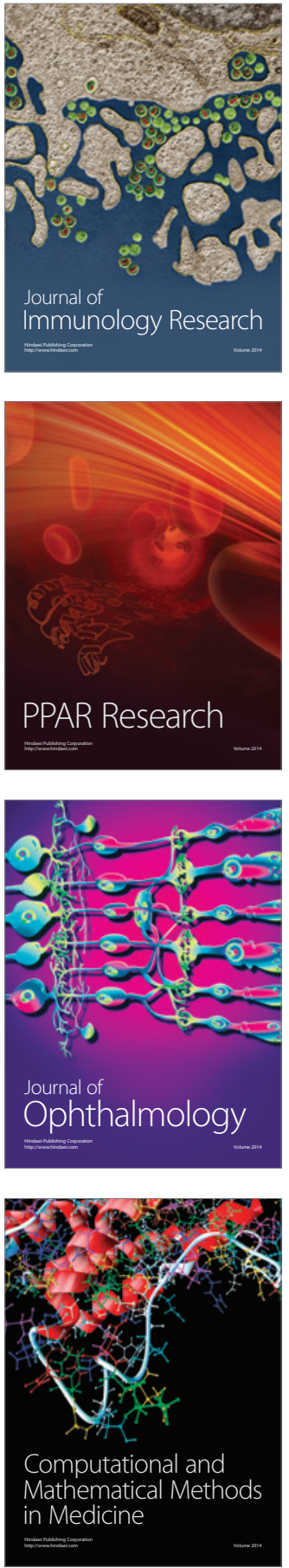

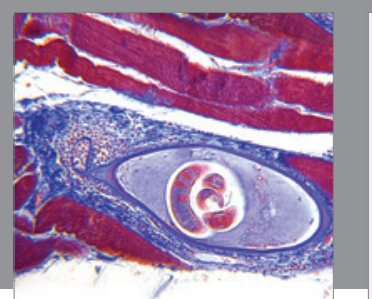

Gastroenterology Research and Practice

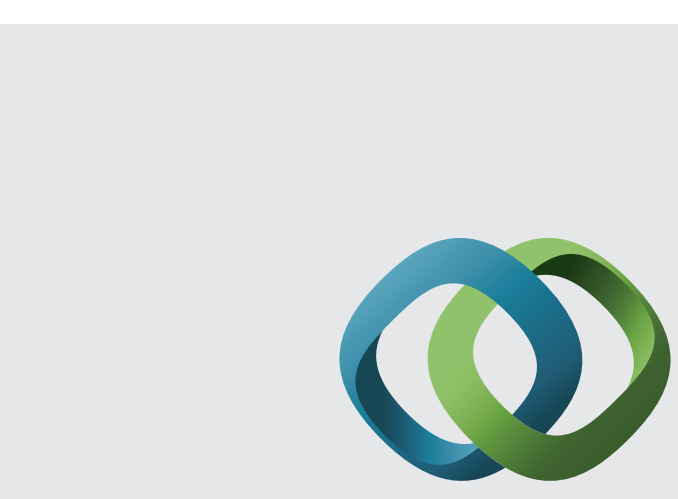

\section{Hindawi}

Submit your manuscripts at

http://www.hindawi.com
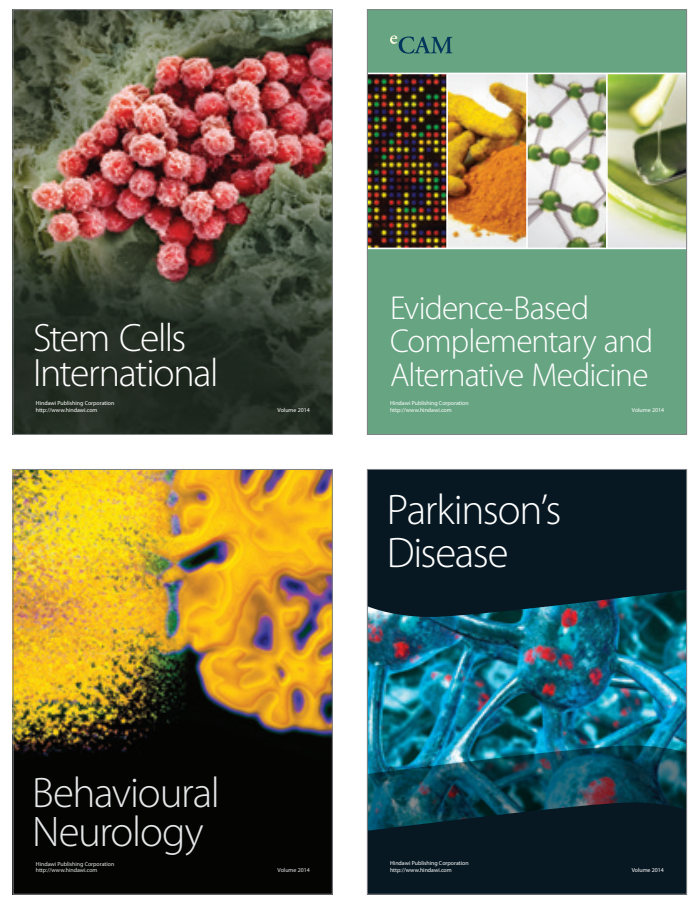
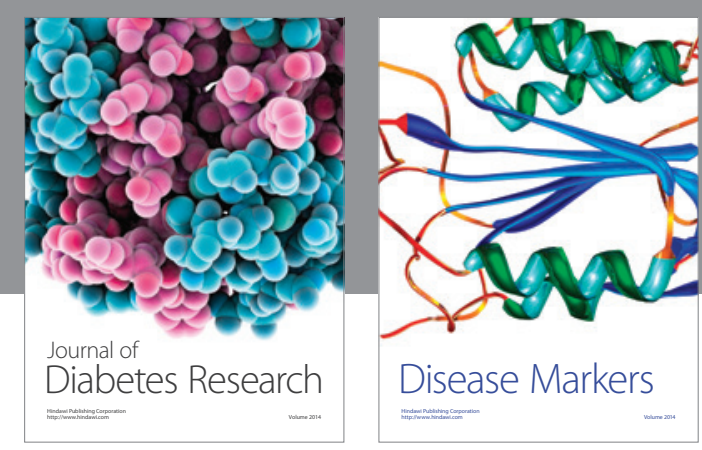

Disease Markers
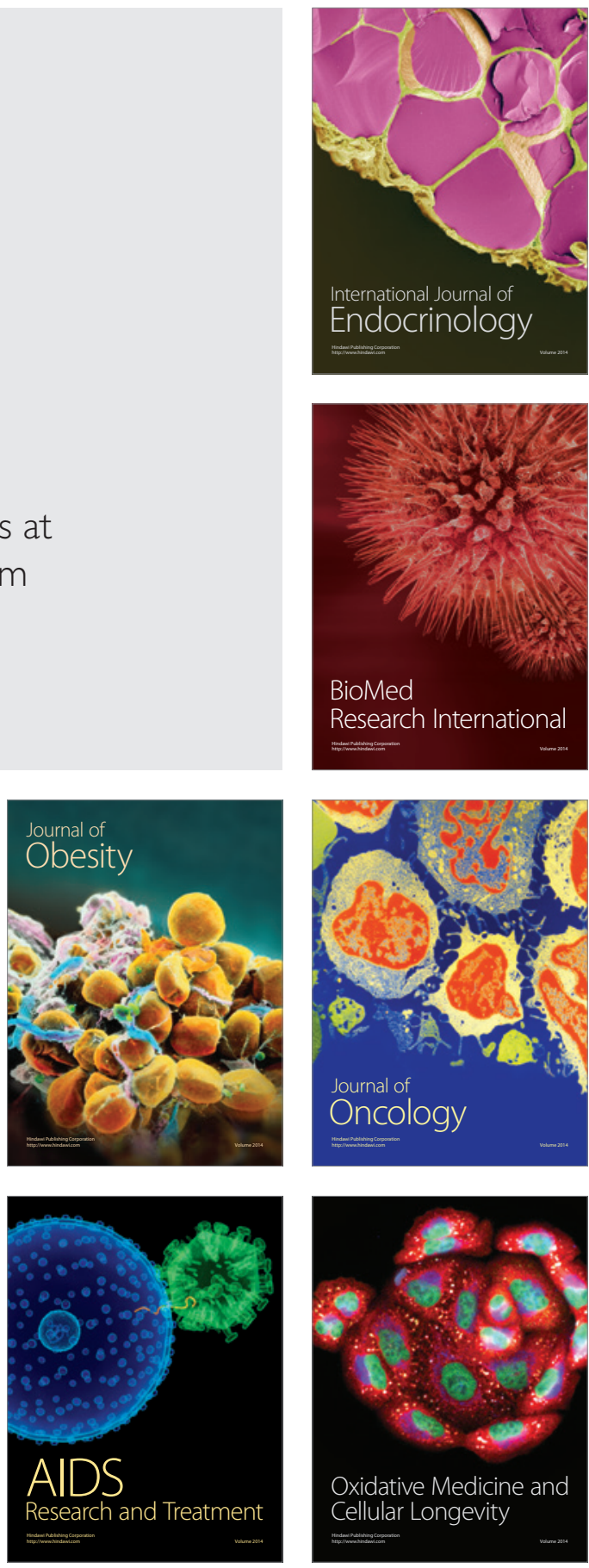